\title{
Fixer le nom de l'ancêtre (Porto-Novo, Bénin)
}

Fixing the Ancestor's Name (Porto-Novo, Benin)

\section{Marie-Josée Jamous}

\section{OpenEdition}

\section{Journals}

Édition électronique

URL : http://journals.openedition.org/span/1390

DOI : $10.4000 /$ span. 1390

ISSN : 2268-1558

\section{Éditeur}

École pratique des hautes études. Sciences humaines

\section{Édition imprimée}

Date de publication : 1 décembre 1994

Pagination : 121-157

ISSN : 0294-7080

\section{Référence électronique}

Marie-Josée Jamous, «Fixer le nom de l'ancêtre (Porto-Novo, Bénin) », Systèmes de pensée en Afrique noire [En ligne], 13 | 1994, mis en ligne le 18 février 2014, consulté le 30 avril 2019. URL : http:// journals.openedition.org/span/1390 ; DOI : 10.4000/span.1390 


\section{FIXER LE NOM DE L'ANCÊTRE \\ (Porto-Novo, Bénin)}

\section{par}

\section{Marie-Josée Jamous}

A Porto-Novo', chaque quartier, occupé par un patrilignage, est divisé en un certain nombre de maisons qui se regroupent autour du sanctuaire de l'ancêtre fondateur, le yox $\jmath^{2}$. Cette disposition est plus

1 Porto-Novo est une ancienne cité-royaume située au sud de la République du Benin. Fondée vers 1730 par des lignages Adja et Aladanu, rejoints par des Tori, Peda, Seto, Ouemenu, etc., la ville de Porto-Novo est le creuset de la culture gun (deux tiers de la population) à laquelle se sont ajoutés des Yoruba. Notre enquête (1967-68) a porté principalement sur un quartier gun, celui du lignage Dravonu, originaire de Ge Dravo, plateau d'Allada. Les Dravonu sont dits avoir transmis les coutumes funéraires d'Adja; ils sont chargés des funérailles royales à Porto-Novo (cf. Pineau-Jamous 1986: 549).

Je remercie $R$. Jamous pour l'aide qu'il m'a apportée pour la rédaction de cet article.

2 Les quartiers les plus importants sont divisés en sous-quartiers correspondant chacun à un segment de lignage centré sur le temple de son ancêtre fondateur. L'ancêtre fondateur du lignage occupe une position prééminente en tant que père et aîné de tous les autres (cf. Pineau-Jamous, 1986). C'est près de son sanctuaire que se déroulent les principales cérémonics de transformation des morts en ancêtres. Nous traduisons par sanctuaire le terme yoxo (de yo = "tombe" et $x o=$ "chambre"), qui signifie littéralement "chambre de la tombe" mais d'une tombe qui ne contient pas le corps. Ce terme s'oppose à yodo, "trou de la tombe", lieu de l'ensevelissement du mort dans sa propre chambre. Le sanctuaire est un édifice tantôt rond, tantôt

Le deuil et ses rites III Systèmes de pensée en Afrique noire, 13, 1994 
qu'une simple occupation du sol, une délimitation d'un territoire; elle inscrit dans l'espace les relations entre les vivants, les morts et les ancêtres et renvoie à un lieu et un temps de l'origine reproduits dans le quartier sous la forme du temple du henuvodun ${ }^{3}$. C'est dans les rites et notamment les rites funśraires que ces relations se manifestent pleinement.

Les premières et secondes funérailles ont certes pour objet d'agir sur différentes composantes des défunts pour les séparer des vivants et les renvoyer dans le pays des morts. Mais tout ce processus de transformation ne se résume pas simplement par la disparition de ces morts. Ceux-ci doivent passer du statıt de défunt à celui d'ancêtre, c'est-àdire de cadavre enterré dans la maison à un nom planté dans le sanctuaire de l'ancêtre fondateur, participant ainsi à la vie du quartier lors du choix du conjoint, lors de la recherche de l'ancêtre protecteur du nouveau-né, lors des fêtes des prémisses, etc. C'est ce passage, qui s'étale dans le temps et dans l'espace, que l'action rituelle des vivants et notamment celui de l'officiante principale, la tanyinon, la prêtresse des ancêtres ${ }^{4}$, doit accomplir.

rectangulaire, qui a été élevé en l'honneur de l'ancêtre fondateur et qui porte son nom. Il contient son asẽ, sorte de parasol-autel, qui le représente, et les asẽ des morts de sa lignée. Cet édifice s'ouvre sur une cour où l'on célèbre les rites saisonniers en l'honneur des ancêtres.

${ }^{3}$ Le pays de l'origine est celui où se rejoignent le monde humain, le monde naturel et le monde cosmique: selon les mythes d'origine, le premier ancêtre est soit descendu du ciel, soit sorti de la terre, ou au contraire, sans passer par la mort, a rejoint le ciel par une corde ou bien s'est enlisé en se métamorphosant en pierre, termitière, arbre, etc. Ce premier ancêtre est le hẽnuvodun du groupe, celui qui a dicté les interdits. Il a son temple dans le quarticr, ses initiés, ses officiants, et fait l'objet de rites saisonniers. (cf. Pineau-Jamous, 1986: 547-548). Ce temple se distingue du sanctuaire de l'ancêtre par sa structure plus complexe. Il abrite une famille de divinités regroupées autour du hẽnuvodun et un lieu d'initiation.

4 Tanyinon est composé de tanyi, "tante paternelle" et non, "mère"; il signifie littéralement "mère des tantes paternelles". Il existe une tanyinon principale par lignage, celle liée à l'ancêtre fondateur de tout le groupe. D'autres lanyinon de moindre importance peuvent être liées au sanctuaire des ancêtres fondateurs de segments de lignage. La tanyinon principale est toujours choisie parmi les vieilles tantes paternelles de la lignée aînée, revenues vivre dans leur maison d'origine. En tant que prêtresse de l'ancêtre fondateur, clle a un statut aussi élevé que celui du chef du lignage, hẽnugan ou xweduto, dont l'une des fonctions est de gérer les biens de 
Mais ce retour du mort sous la forme d'un nom et d'un autel qui manifestent sa nouvelle relation avec les vivants n'est possible qu'après un long processus rituel qui s'étend sur plusieurs années. Au cours des premières et secondes funérailles, l'action rituelle des vivants consiste à la fois à effacer toute trace des défunts, couper toute relation avec eux, et à leur reconstruire une identité au pays des morts.

\section{Les premières funérailles 5}

Les premières funérailles sont une affaire familiale; les membres de la maison du mort et les gendres occupent les rôles cérémoniels importants. Elles s'étendent sur neuf jours et se déroulent essentiellement à l'intérieur et autour de la maison où le mort sera enseveli. Tout défunt doit être enterré sur le lieu où il est né ${ }^{6}$. Cela est valable non seulement pour un homme mais aussi pour une femme. En effet, à sa mort, une femme mariée sera reconduite dans la maison de son père ${ }^{7}$. De plus, au moment de sa mort, le défunt est dit être retourné dans son pays d'origine, celui plus lointain de l'ancêtre vodun du clan (Gé pour les Dravonu, Aja pour les rois de Porto-Novo). Ainsi la mort

l'ancêtre et de veiller au respect de ses interdits. Ces deux personnages, qui sont intronisés devant le sanctuaire de l'ancêtre fondateur, ont des rôles complémentaires au moment des secondes funérailles.

5 Il existe une seule expression pour désigner les funérailles dans leur ensemble : cis $n u$ (cis = "mort, cadavre"; $n u=$ "chose"). Elle est utilisée aussi bien pour les premières funérailles que pour les secondes.

${ }^{6}$ Le transfert d'un défunt de l'endroit où il est mort à sa maison d'origine est accompagné de chants (cf. Guédou, 1985: 507-508).

${ }^{7}$ A Porto-Novo, toute fille du lignage doit être enterrée dans la maison de son père ou de son frère où une chambre lui est réservée. En effet, une fille mariée n'est jamais coupée de son lignage, elle revient pour les rites à son ancêtre et au vodun lignager, en particulier si elle fait partie de ses initiés (vodunsi). Après la ménopause, elle s'installe définitivement dans la maison paternelle où elle remplit pleinement son rôle de tante paternclle qui a autorité sur les enfants de ses frères. Avec la tanyinon, les tantes paternelles sont au centre de la vie rituelle du lignage. 
est agie et pensée en termes de retour et plus généralement de parcours. Les premières funérailles se déroulent en plusieurs étapes ${ }^{8}$.

\section{Ensevelir le mort avec de nouvelles enveloppes pour le pays des} morts

La première cérémonie ne dure pas plus d'un ou deux jours. Elle se déroule dans la chambre du mort ${ }^{9}$. Les proches parents procedent à la toilette de celui-ci, accompragnés des orphelins qui versent l'eau. Les orifices du corps sont bouchés. Les cheveux sont rasés, les ongles coupés et déposés dans une calebasse qui sera enterrée avec le mort $^{10}$. On expose le mort sur un canapé en bambou couvert d'une natte kplakpla (natte faite avec des cours de tiges de palmier a raphia) ${ }^{11}$. Le corps est recouvert de la tête aux pieds d'un linceul blanc et de beaux pagnes de velours donnés par les orphelins, les gendres et les amis ${ }^{12}$. Pendant ce temps, les gendres et amis creusent la tombe dans la chambre du mort ${ }^{13}$ dans une ambiance très joyeuse avec de la musique, des chants et des boissons alcoolisées.

La forme de la tombe varie selon les lignages ${ }^{14}$. On dépose sur le fond un canapé en bambou recouvert d'une natte kpakpla ou simplement une natte kpakpla. Le mort est enterré dans la position du fœtus,

${ }^{8}$ Nous décrivons ici les funérailles d'un homme d'âge mur ayant marié ses enfants. Les rites pour les hommes et les fernmes différent peu. Les initiés du vodun font l'objet de rites particuliers dont nous ne parlerons pas ici. Les premières funérailles des mauvais morts, ceux dont la mort accidentelle est attribuée à un vodun, sont pris en charge par le prêtre de ce dernier (Kiti, 1986: 10-12).

${ }^{9}$ Les rites des premières et secondes funérailles ont été observés lors de notre séjour à Porto-Novo en 1967-68. Cependant nous n'avons pas assisté à l'ensevelissement; la description de cette partie du rite est faite d'après les informations recueillies.

10 Quand le mort est décédé à l'étranger ou enterré dans un cimetière, cette calebasse, à laquelle on ajoute du sable du dessus de la tombe, est enfouie dans la chambre. Selon Maupoil (1986: 1397), cheveux, rognures d'ongles et sable représentent l'âme du mort, son $y \varepsilon$, son ombre.

11 La natte kplakpla est une natte de fabrication grossière par opposition aux nattes bien finies dont on se sert dans la vie quotidienne.

12 Aujourd'hui on habille le mort des plus beaux vêtements qu'il possédait.

13 Si le mort est un enfant, il est enterré derrière la chambre de son père.

14 Voir Akindélé et Aguessy, 1953: 99-100. 
la tête tournée vers l'océan. Les gendres, les amis et les orphelins font placer sur le mort des pagnes neufs (les uns doivent lui permettre de tenir son rang au pays des morts ${ }^{15}$, les autres sont envoyés par son intermédiaire aux anciens morts de la famille), des bouteilles de boissons alcoolisées (pour permettre au défunt de boire dans l'autre monde), des cauris et des pièces de monnaie (pour payer le passeur dans l'au-delà). Au moyen de bambous coupés, on ferme l'ouverture de la tombe et on étend au-dessus une natte kpakpla. Le tout est recouvert de terre friable extraite de la tombe.

\section{Braler les "enveloppes" proches du mort et aplanir la tombe}

La deuxième cérémonie se déroule le troisième jour (le matin très tôt). Elle se nomme ags vive. Le terme ago a une signification complexe. $A g$ ost le nom des rites où l'on traite les restes du mort. Il désigne aussi les morts sous leur aspect de restes actifs et dangereux, restes qui prennent des formes différentes dans chaque contexte rituel. Ici il s'agit principalement de vêtements, d'effets personnels du mort que nous appellerons en suivant Akindélé et Aguessy (1953: 109) l'enveloppe du mort ${ }^{16}$. Selon Guédou (1985: 249-251), vive signifie "bile" mais aussi "colère", "douleur", "précieux" ou "proche". S'agit-il d'apaiser la bile du mort, sa colère, ou bien la douleur des

15 Selon Herskovits (1967: 1, 373), les vêtements donnés par les enfants pour couvrir le corps dans la tombe sont appelés "vêtements tués pour la tombe" (I: 373 ). Dans un chant recueilli par M. Quenum (1983: 125-127), on oppose le fils qui a honoré son père mort en le recouvrant d'une étoffe de soie, d'une blouse et d'un beau chapeau, à celui qui l'a enterré sans joindre tous ces objets. Ce dernier sera repoussé par son père dans le pays des morts: "va-t'en loin de moi, que viens tu faire ici, fils de chien". Au contraire, le premier sera bien reçu: "viens, ô fils que j'idolâtre, viens et sois héros". Les mêmes chants associent l'envoi des habits et l'offrande d'alcool.

16 Selon Guédou (1985: 231), c'est agbaza, la "peau" qui va dans la tombe. Le terme aghaza dénote les qualités et les défauts (couleur, résistance, rugosité, douceur) du corps par opposition au terme wutu qui désigne le corps en tant que siège et autel de l'être. On peut faire l'hypothèse que l'enveloppe est une extension de la peau. Tous les vêtements du mort sont dangercux à porter (sauf par les enfants de fille), cependant il faut distinguer entre les vêtements quotidiens prockes du mort détruit par le feu et ceux d'apparat marquant son statut social, qui seront brûlés à la fin des secondes funérailles pour être envoyés au pays des morts. 
deuilleurs? Il semble que le mot joue sur ces deux registres. Ce sont à la fois les affects du mort et ceux des deuilleurs qu'il faut traiter en brûlant les effets proches du disparu qui étaient partie intégrante de sa personne durant sa vie (son enveloppe). Dans un coin de la palmeraie, hors de la ville, des parents brûlent la natte sur laquelle il couchait, les vêtements dans lesquels il est mort et les ustensiles dont il se servait quotidiennement. On revient avec $a z a^{17}$ (feuille de palmier) et akpa (côté plat de la tige du palmier). Au retour, un nonuvi ("enfant de fille") aplanit la tombe en frappant avec akpa ${ }^{18}$. Les parents jettent du sable sur la tombe en disant: boyils, "qu'il parte loin" (yils veut dire "va loin") ou bozomils, "devance-moi". Ils tournent autour de la tombe en récitant des chants funéraires (neuf pour un homme, sept pour une femme). Par ce rite d'expulsion, on écarte provisoirement le danger que représente le mort et on apaise la douleur des vivants.

\section{Purifier et affermir la maison}

Le lendemain d'ags vive, les jeunes gens purifient la maison (xwe kpikpo) avec aza et akpa ramenés de la brousse. Ils se précipitent dans la maison en criant: "que le mort sorte et fasse place au vodun" (Age! Kuton, hun bio xwe, ya a). La feuille de palmier (az $\tilde{a})$ est traînée dans toutes les cases qui sont aspergées avec de l'eau où ont macéré des feuilles liturgiques contenue dans une poterie. Les officiants frappent ensuite le sol avec akpa et disent: "ag J est sorti, le vodun est rentré" (agy ton hun bio xwe). Ce rite est aussi appelé: "il faut affermir la maison" ( $E$ ni ni xwe).

Au moment de la mort, la maison est considérée comme dévastée: "le feu est tombé sur le toit", "elle est noyée". Elle est en quelque sorte coupée du reste du quartier. Purifier la maison, c'est aussi

${ }^{17}$ Porter azã, c'est supplier pour se faire pardonner, c'est aussi se protéger contre un danger (Herskovits, 1967: 1, 214).

18 "Les enfants de filles" jouent un rôle important pendant les funérailles. On dit qu'ils chassent le malheur. 
l'affermir, c'est-à-dire rétablir sa relation avec les autres maisons du quartier et le temple du vodun ("ags est sorti, le vodun est rentré") 19.

\section{Manger, boire et danser avec le mort}

Le neuvième jour après l'enterrement, la tanyinon, prêtresse de l'ancêtre fondateur, fait une libation au yoho pour prier les ancêtres de venir assister à la cérémonie, d'éloigner les querelles et d'accueillir le mort. Elle verse de l'eau et du sodabi (alcool local) sur la tombe (à l'emplacement de la tête). Ce jour est l'occasion d'une grande veillée funéraire qui se passe à l'extérieur de la maison, sur une petite place. Les gendres et les fils du disparu doivent tuer des porcs et les cuisiner pour nourrir les invités, et leur offrir des boissons alcoolisées. Ils louent les services des orchestres funéraires dits zêli pour animer les danses de la veillee. On dit que l'on danse, que l'on fait ripaille avec le mort ${ }^{20}$. Cette cérémonie finale est appelée medo alisa, "recréation du défunt". C'est une première mise en scène de la réunion du défunt avec ses ancêtres dans le pays des morts et de leur réjouissance avec et à travers les vivants. Ce même rite se reproduira lors des secondes funérailles.

\section{Remarques:}

- Le traitement du cadavre

Il se fait essentiellement dans la maison du mort, la maison où il est né. Dans les chants recueillis par Guédou, au moment de mettre le

19 Il semble qu'il existe une sorte d'incompatibilité entre la mort et le vodun. Celui-ci est un héros-ancêtre qui s'est transformé sans passer par la mort. Lorsqu'une des initiées meurt, on doit la séparer de son vodun par des rites appropriés. Enfin il n'est pas bon que les initiées du vodun entrent dans une maison mortuaire.

${ }^{20}$ Chez les Fon, le mort était représenté autrefois par un simulacre constitué d'un pagne, offert par les orphelins, enroulé dans un natte (cf. Herskovits, 1967: 1, 371). Nous avons assisté à une cérémonic où le mort était représenté par unc photo de lui-même en vêtement d'apparat. 
cadavre dans la tombe, l'expression suivante revient comme un refrain: "je retourne dans le giron maternel" (Guédou, 504) ${ }^{21}$.

L'inhumation des morts dans la maison d'origine, celle où l'on est né, en fait une propriété inaliénable ${ }^{22}$. Ainsi les morts inscrivent chaque maison du quartier dans la pérennité et marquent son extension et ses limites. Le quartier lui-rnême est considéré comme une maison (xwe), dont la partie la plus sacrée est la "tête de maison" (xweta), maison aînée où se trouve le sanctuaire de l'ancêtre fondateur. C'est là que l'asẽ du mort sera planté à la fin du processus d'ancestralisation, là où la tanyinon appelle les ancêtres à l'accueillir.

Des lieux et parcours extérieurs au quartier et à Porto-Novo sont évoqués. Le défunt est dit être retourné au lieu d'origine de son clan, celui d'où est venu l'ancêtre fondateur du quartier. Il fait donc le chemin inverse de cet ancêtre ${ }^{23}$. Le mort enterré est orienté vers l'océan, vers le pays des morts qui se situe au delà de la mer. Un parcours est effectué par les vivants dans la brousse pour brûler les restes du mort.

\section{- Le traitement des enveloppes}

Les pagnes neufs sont enterrés avec le défunt pour constituer sa nouvelle enveloppe dans le pays des morts. Par contre, ses vêtements quotidiens et ses objets usuels ont été brûlés loin de la maison, dans la brousse. On saisit l'importance de l'enveloppe (en même temps habit et objet) qui fait partie de la personne vivante ou morte. Il ne suffit pas de détruire celle qui relie le mort au monde des vivants: il faut lui en

21 Il ne s'agit pas de la maison maternelle. mais de celle du père, où la mère a accouché.

22 Il existe des émigrés qui ont acheté des maisons à Cotonou. Au moment de leur mort, leur corps est enterré dans la maison d'origine qui a un statut différent de celle de Cotonou, laquelle peut être vendue sans aucun problème.

23 Bourgoignie (1972: 105) décrivant la pérégrination d'un mort à partir d'un village lacustre où il n'avait pu être enterré faute de place, souligne que "l'itinéraire suivi était en quelque sorte l'inverse de celui emprunté par les ancêtres". 
constituer une nouvelle pour lui permettre de tenir son nouveau rôle dans le monde de l'au-dela 24 .

\section{- La relation mort/vivants}

C'est la famille proche qui prend en charge l'inhumation du mort, première étape de la séparation du défunt des vivants. On se débarrasse de la souillure du mort en brûlant ses vêtements (ag.) vive), en purifiant la maison, en rasant les deuilleurs. Mais cela ne suffit pas, la souillure du cadavre est circonscrite, prise en charge principalement par les veuves jusqu'à l'extraction du crâne $\mathrm{e}^{25}$. D'autre part, le renvoi du mort nécessite de préparer sa place dans l'au-delà. Ce sont les fils et les gendres ${ }^{26}$ qui s'en chargent par leurs prestations en vêtements, cauris et boissons. Mais il faut aussi se réjouir avec le mort ici-bas; là encore ce sont les fils et les gendres qui fournissent la nourriture, les boissons et les orchestres nécessaires au rite qui clôt ces premières funérailles.

24 Il faut signaler l'importance des vêtements: prestation de pagnes lors de la déification des ancêtres et dans tous les rites mettant en scène le retour des morts, en particulier dans les familles royales d'Abomey (voir la description des rites des nesu xwe dans Herskovits, 1967: I, 220). Chez les Yoruba du Nigéria, lors du rite des Egun, les morts reviennent sous forme de magnifiques vêtements que les vivants ne doivent pas toucher (photos et description dans Verger, 1954: 155-158). Selon Glele (1974: 66), chaque année le roi d'Abomey devait procéder à un bain rituel de purification et de régénération à la suite duquel il s'habillait de vêtements tissés avec du fil de coton de l'année et se débarrassait de ses habits royaux que revêtait un jeune garçon que l'on faisait disparaître.

25 Le poids et la durée du veuvage sont beaucoup plus importants pour une veuve (trois à neuf mois) que pour un veuf. Celui-ci, en tant que gendre de la maison où sa femme est enterrée, doit participer aux prestations funéraires. Ses interdits de deuil sont très réduits: ne pas avoir de relations sexuelles jusqu'à la fin des premières funérailles (Tidjani, 1952: 19-20).

26 Les gendres ont des obligations plus ou moins importantes en fonction de leur relation avec le mort ou la morte. La belle-mère, le beau-père sont ceux pour lesquels on doit faire le plus de dépenses. Les frères et les scurs du beau-père, en particulier ceux qui ont participé aux discussions avant le mariage, ont droit aux mêmes honneurs. 


\section{Intervalle entre les premières et les secondes funérailles}

\section{Le deuil des veuves}

Le deuil des orphelins ne se manifeste pas dans la vie quotidienne. Cependant ils doivent prendre part au repas sacrificiel au moment de l'extraction du crâne de leur père ou de leur mère et lors de la cérémonie qui clôt les secondes funérailles avant de pouvoir participer à des cérémonies du même genre pour des parents plus éloignés. Par contre, le deuil des veuves est beaucoup plus marqué et dure neuf mois, jusqu'à l'extraction du crâne du défunt. Elles ne doivent pas sortir du quartier, ni aller au marche; elles portent des habits sombres et ne coiffent pas leurs cheveux. Il leur est interdit d'avoir des relations sexuelles $^{27}$.

\section{L'extraction du crâne}

C'est la première cérémonie ayisun ${ }^{28}$. Elle se fait dans chaque famille, dans chaque maison qui a eu un décès: en principe sept lunes après la mort d'une femme et neuf lunes après celle d'un homme. Un oncle paternel de la maison ouvre la tombe, décolle le crâne et le lave avec de l'eau liturgique où ont macéré des feuilles ${ }^{29}$. Le crâne est ensuite exposé sur des feuilles de la même espèce. La tante paternelle de la maison sacrifie un poulet et verse le sang sur le crâne $e^{30}$. Le poulet est grillé et mélangé avec de l'huile de palme. La tante paternelle offre une part de cette nourriture au crâne puis tous les membres

${ }^{27}$ Selon Bourgoignie (1972: 306), chez les Toffinu la charge du deuil est confiée aux veuves, aux filles et aux nièces. Selon mes informateurs, les veuves portaient autrefois un pagne de raphia, semblables à la natte kplakpla. Est-ce un signe de l'identification de la veuve au mort?

28 Ayisun est le nom de l'ensemble des rites qui ont pour objet le traitement des crânes des défunts. Selon Akindele et Aguessy (1953: 108), ayi = père et sun = coutume. Il faut noter qu'ayi signifie terre dans le sens de terre nourricière, objet de vénération, et se distingue de $k a$ qui désigne l'argile ou le sable, matière première du corps. Sun désigne la coutume au sens d'interdit, d'obligation.

${ }^{29}$ Ces feuilles appelées deseregue ou deserema sont aussi utilisées pour laver la tête des initiés.

${ }^{30}$ Une cérémonic analogue se déroule au début de l'initiation au vodun. 
de la maison se partagent les restes (on en garde pour les absents). Manger ces restes marque la fin des interdits pour les orphelins, qui peuvent désormais assister à des cérémonies du même type dans d'autres familles. Le lendemain, le crâne est recouvert de toile blanche et de beaux pagnes multicolores, l'ensemble ayant une forme conique. Les parents et amis viennent le saluer comme pour fêter le retour d'un voyageur $^{31}$. La tête du poulet sacrifié a été remise à un parent du défunt qui doit en manger la chair sans casser l'os. Le crâne de l'animal est conservé. Le soir, on apprête un couffin (ad.skpo), on y place le crâne du mort enveloppé dans une toile blanche et on met le crâne du poulet dessus. On ferme solidement le couffin qu'on accroche au mur de la chambre du défunt. Quand le mort a été enterré au cimetière catholique ou protestant, le crâne est remplacé par un petit pot appelé meyize $e^{32}$ qui fait l'objet des mêmes rites.

A la fin de cette cérémonie, on laisse pourrir le corps sans tête dans la maison paternelle, alors que le crâne est préparé pour son départ dans l'au-delà qui s'effectuera lors des secondes funérailles.

\section{Fin de deuil}

Après la cérémonie d'extraction du crâne a lieu celle du da lun lun qui marque la fin du deuil des veuves. On leur rase la tête et les interdits sont levés. Elles se parent de nouveaux habits, peuvent de nouveau aller au marché. Un grand repas est offert aux vieilles tantes paternelles. Les veuves sont attribuées par la tanyinon aux cadets de leur époux devant le sanctuaire de l'ancêtre. Le nouveau mari doit contribuer au paiement du repas de sortie de deuil ${ }^{33}$.

${ }^{31}$ La salutation adressée est la suivante: mi ku afo juba. Mi ku signifie "je salue", et afi odju ba "celui qui a vu". P. Verger et J. Braga nous ont signalé qu'ils avaient assisté à un rite analogue durant lequel le crâne paré avait été promené à travers le marché. Le retour du voyageur est ainsi rendu public (communication orale).

${ }^{32}$ De $m \varepsilon$ qui signifie "individu", $y i$, "prendre" et $z \tilde{e}$, "pot". Mcyizẽ veut done dire "le canari qui prend l'individu". Selon Adoukonou (1979: 283), $m \varepsilon$ fait référence au personnage social tel qu'il se constitue dans son propre devenir, de même que le nyi, le "nom" que l'on s'attribue dans les différentes périodes de sa vie.

${ }^{33}$ Il doit offrir les aliments préférés du défunt (Bourgoignie, 1972: 307). 
Il est intéressant de mettre en parallèle la transformation du mort durant ces premières funérailles et le deuil des veuves. Pendant neuf mois, période durant laquelle le corps du défunt pourrit dans la tombe, les veuves doivent accompagner cette décomposition, cette putréfaction, sans sortir pratiquement de la maison, sans se laver, sans changer d'habits, laissant leurs cheveux ébouriffés. L'extraction du crâne et la sortie du deuil sont concomitantes. Le crâne qui représente le corps bien cuit du mort, est lavé, habillé de beaux pagnes; les veuves ont la tête rasée, sont parées et peuvent de nouveau circuler hors du quartier. Mais ce qui caractérise aussi ce moment, c'est la séparation du destin du défunt d'avec celui de sa veuve: le crâne est préparé pour son départ vers l'au-delà, le chemin de l'ancestralité est tracé et s'accomplira lors des deuxièmes funérailles; la veuve, après avoir accompagné son époux défunt, reprend son rôle social parmi les vivants, retrouve sa place au marché et peut se remarier et assurer son rôle de procréatrice.

\section{Les secondes funérailles}

Si les premières funérailles sont familiales et concernent essentiellement le traitement d'un cadavre, les deuxièmes funérailles font intervenir tout le quartier, tous les membres du lignage et concernent plusieurs morts dont on va traiter collectivement le crâne et le souffle, c'est-à-dire ce qui reste du mort après que le cadavre a pourri dans la tombe $\mathrm{e}^{34}$.

Avant que ces cérémonies puissent commencer, il est nécessaire que la paix règne dans chaque famille et dans tout le quartier. On règle les querelles ${ }^{35}$. On va boire de l'eau de la source zekpon (située dans la banlieue nord de Porto-Novo) ou bien on va chercher un peu de

34 Ces secondes funérailles concernent aussi les mauvais morts dont on a racheté les crânes au détenteur du vodun qui les a tués (prêtre du Xevioso pour ceux qui ont été tué par la foudre, prêtre du gu pour ceux qui ont été tués par le fcu, ctc.).

35 Les plaintes en justice qui résultent des conflits entre membres du quartier doivent être retirées. 
cette eau que les membres de chaque maison boivent devant le xweli, le vodun familial, placé au centre de la cour de la maison. Ce rite doit expulser toutes les mauvaises intentions qui sont à l'origine des disputes ou des actions de sorcellerie. Il arrive souvent que, lors des secondes funérailles, une offrande soit refusée par un mort. Il faut consulter le $f a$ pour savoir quelle querelle entre les vivants empêche le mort d'accepter ${ }^{36}$. Le $f a$ dicte l'offrande ou le sacrifice qui autorisera la reprise des rites.

Ces secondes funérailles sont inscrites dans le calendrier saisonnier et dans le cycle agricole. Elles se déroulent au milieu de la saison sèche, quelque temps après la récolte de l'igname et les offrandes des prémisses aux ancêtres et au vodun. On consulte le fa dans le sanctuaire de l'ancêtre fondateur du quartier pour fixer la date des cérémonies. C'était autrefois une fête annuelle suivie immédiatement du hunhue, de la grande fête du vodun ${ }^{37}$ puis de nouvelles semailles. Les secondes funérailles se déroulent actuellement tous les deux ou trois ans, et sont toujours suivies par la fête du vodun. Elles concernent tous les morts de la période ${ }^{38}$.

Quatre séquences importantes sont à distinguer: 1) la cérémonie collective ayisun qui concerne les crânes, 2) la cérémonie cis wiwo qui traite le souffle, "l'âme" des morts, 3) la cérémonie ags durant laquelle on doit brûler les enveloppes des morts, c'est-à-dire tous leurs vêtements et les objets qui leur ont appartenu, enfin 4) la cérémonie asennyidote qui est l'installation du parasol, autel du mort, auprès de l'ancêtre fondateur, dans le sanctuaire de ce dernier.

\footnotetext{
36 Il peut s'agir aussi d'une querelle non réglée avec un mort.

37 C'est à la fin de cette fête que l'on choisit de nouvelles initiées pour le henuvodun.

38 Il faut signaler que cette périodicité observée chez les Dravonu de Zebu Aga ne concerne pas tous les lignages de Porto-Novo. Les familles royales n'accomplissent ces rites qu'au bout d'une dizaine d'années.
} 
1. La cérémonie collective ayisun ${ }^{39}$

Elle commence le jour du marché de $\operatorname{Djegan}^{40}$ et dure neuf jours. Elle se déroule pour l'essentiel devant le sanctuaire de l'ancêtre fondateur et dans une salle adjacente appelée ayisunto. Le lignage est représenté par son chef, le xweduto, et chaque segment doit être représenté par ses mexo, ses "anciens", et ses tanyi, ses tantes paternelles. Les officiants principaux sont la tanyinon, la prêtresse de l'ancêtre fondateur, qui préside tous les rites, et un sithuto, personnage qui est chargé de laver les vodun et ici les crânes des morts.

- Rassembler et laver les crânes des morts

La première cérémonie commence à la tombée de la nuit et se déroule jusqu'à l'aube. Chaque famille apporte le couffin adskpo contenant le crâne du défunt ainsi que deux ayisuzẽ , deux pots en terre cuite (un grand et un petit). On ajoute un meyizen, un pot représentant les crânes des morts qu'on aurait oubliés. Deux grandes jarres sont placées devant le sanctuaire de l'ancêtre, l'une contenant uma (de l'eau tiede mélangée à des feuilles liturgiques), l'autre de l'eau de pluie. Un lettré, une liste à la main, appelle les défunts de chaque maison ${ }^{41}$. Le sîhuto, le purificateur, reçoit chaque crâne et le baigne dans l'eau tiede uma disant: "Un tel, nous te baignons comme nous l'avons fait pour tes ancêtres, viens au milieu des tiens, sois le bienvenu". Il le baigne ensuite dans l'eau de pluie et le remet aux parents qui s'alignent devant la salle d'ayisun. Un grand trou est creusé pour recueillir l'eau du bain afin qu'elle ne ruisselle pas car elle véhicule la souillure du mort. Il

${ }^{39}$ La cérémonie d'cryisun d'un chef de lignage a lieu neuf jours avant celles des autres morts. Elle se passe dans sa propre maison, où son crâne est honoré avec celui d'un enfant. Si cet homme a reçu un titre de dignitaire auprès du roi, son crâne sera enterré dans sa cour en attendant que l'on érige un sanctuaire à son nom marquant ainsi une nouvelle segmentation du lignage et du quartier.

40 Il s'agit du premier jour de la semaine traditionnelle de quatre jours.

41 Un lettré est ici une personne qui sait lire et écrire. C'est le Xweduto, chef de lignage, qui a établi la liste; l'oubli d'un mort mettrait sa vie en danger. 
serait dangereux pour les membres du lignage d'y tremper les pieds, en particulier pour les veuves qui n'auraient pas respecté les interdits lies à leur condition.

Un assistant place les crânes sur les feuilles liturgiques ajama disposées par terre. Chaque famille remet à la tanyinon un coq, une poule, des noix de cola et du sodabi. La tanyinon procede au sacrifice puis arrose le crâne avec le sang des victimes en répétant des paroles de bienvenue et des souhaits de prospérité pour le quartier. Chaque crâne reçoit comme offrande des haricots cuits et des poulets grillés arrosés d'huile de palme.

- Mettre les crânes dans des jarres de terre cuite

La tanyinon met chaque crâne dans le petit pot, puis place celui-ci dans le grand pot et le recouvre d'un linge blanc ${ }^{42}$. Elle dispose ces pots sur les nattes kplakpla étendues dans la salle d'ayisun, en prenant soin de ne pas mettre deux anciens ennemis l'un à côté de l'autre (ils pourraient se quereller et l'on retrouverait les pots cassés). Après une libation collective, elle procède au "jeu de cola" pour savoir si les morts sont satisfaits et leur adresse les paroles suivantes: "Voilà, vous êtes exposés, vous êtes assis, nous vous entourons et nous faisons ce que nos ancêtres nous ont enseigné de faire et ce que nous avons fait à nos ancêtres, en conséquence, accordez-nous la paix dans le pays, dans les familles, qu'il y ait beaucoup de richesses, de progéniture, que les enfants présents et absents vivent en bonne santé". Les assistants font un repas collectif avec les restes des sacrifices, boivent, chantent et dansent jusqu'au lever du jour.

- Habiller et exposer les ayisun

Le matin, chaque famille habille richement la jarre de son mort, tenant compte de ses habitudes dans la vie quotidienne. Tous sont revêtus de beaux pagnes neufs, de chapeaux en feutre ou de bonnets

42 Dans le cas de jeunes enfants, plusieurs crânes sont mis dans la même jarre. 
de dignitaires pour les hommes et de turbans et de bijoux pour les femmes. D'autres objets qui plaisaient aux morts sont ajoutés: poupées, colifichets, etc ${ }^{43}$. Les morts ainsi parés sont exposés durant neuf jours.

- Nourrir les ayisun, boire, manger et danser avec eux.

Chaque matin se déroule le rite du réveil, adsfifon. Les morts reçoivent des mets qu'ils aimaient de leur vivant: café au lait, pain grille, omelette, haricots abobo, beignets etc. On leur offre de nouveau un repas à midi puis un autre le soir. Les assistants mangent ensuite les restes de l'offrande, boivent, chantent et dansent tard dans la nuit. Le lendemain, recommence le même cérémonial et cela jusqu'au neuvième jour. Pendant cette période, les parents proches des défunts et leurs gendres apportent des animaux à offrir en sacrifice en l'honneur des morts exposés. Il faut signaler que le cinquieme jour est celui des grandes libations et des sacrifices de coqs, cabris et poulets offerts par toute la collectivité du quartier pour l'ensemble des morts. Le neuvieme jour, les jeunes enfants de la maison et les enfants de filles (ñonuvi) revêtent les habits des morts. Précédés par la tanyinon, accompagnés par les femmes mariées dans les différentes maisons (les yao), ils vont en cortège dans le marché, chantant, dansant et imitant la démarche de ceux qu'ils incarnent et auxquels ils s'identifient dans ces circonstances. Il s'agit de présenter une dernière fois les morts au marché afin qu'ils ne reviennent plus hanter ces lieux ${ }^{44}$.

${ }^{43}$ Kiti donne une bonne description de la cérémonie (Kiti, 1968: 16).

44 Le marché est un lieu important de la vie rituelle dans le Bas Dahomey. A l'opposé des quartiers, c'est un lieu ouvert de rencontres des ethnies et des lignages. Dans les cités-royaumes, il est situé devant le palais royal. D'autres marchés sont situés à des carrefours à l'intérieur des villes, des villages et aussi en brousse. Selon Mondjannagni (1977: 279) "le carrefour est un endroit privilégié pour les morts errants en attendant qu'on organise à leur intention les cérémonies définitives qui les fixent pour de bon dans la sphère des ancêtres divinisés". 


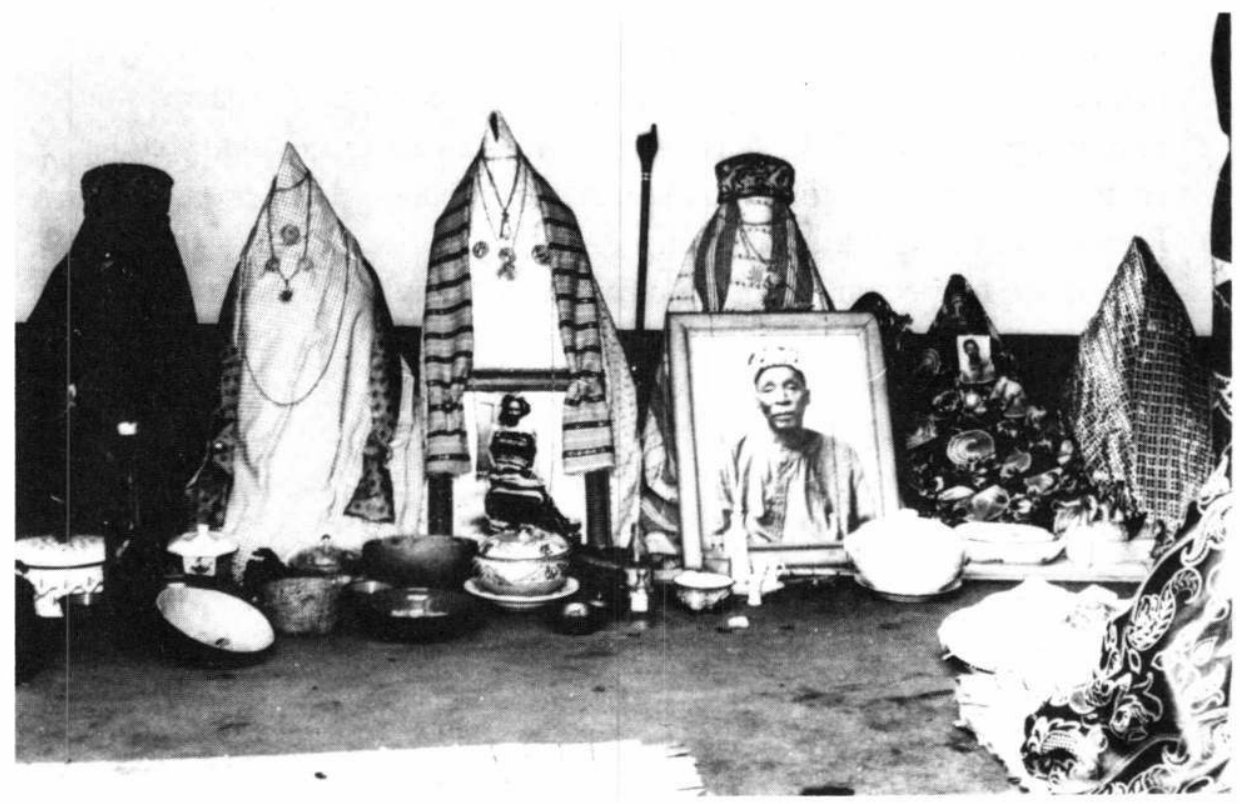

Exposition des ayisun 
- Embarquer les ayisun vers le pays des morts

Le neuvième jour au soir, on offre un dernier repas aux morts ${ }^{45}$. Le sĩhuto creuse une fosse appelée ha kpikpa, "pirogue taillée", derrière le sanctuaire de l'ancêtre. Les vêtements qui enveloppaient les jarres contenant les crânes sont repris par les familles ${ }^{46}$. Pendant ce temps, les femmes préparent les plats suivants: aze-bobo (pâte de pois chiche), li-wo (pâte de gros mil) et $a d o g b o$ (pâte de haricots) dont une part servira à fermer hermétiquement le couvercle de chaque jarre. Le reste des mets est mangé par l'assemblée des assistants. Les jarres sont ensuite alignées dans la fosse par ordre de décès. Le sthuto et les anciens referment la fosse "en secret", en ne laissant aucune trace ${ }^{47}$. Rien ne doit plus marquer ce lieu qui n'est l'objet d'aucun culte. On dit que les morts sont partis en pirogue sur la rivière, vers la mer.

45 A ce moment peuvent avoir lieu des rites de type ordalie avec confession publique des membres du lignage. Il s'agit de boire l'eau qui a servi à laver les crânes en demandant aux morts d'emporter les coupables avec eux.

46 Dans certains lignages, les vêtements sont enterrés avee les pots (Akindelé et Aguessy, 1953: 109), dans d'autres, ils sont repris par les familles ou disputés lors d'une compétition (Kiti, 1968: 17).

${ }^{47}$ Dans les quartiers segmentés en plusieurs sous-quartiers, on consulte le fa pour savoir si le crâne sera embarqué près du sanctuaire de l'ancêtre fondateur ou près de celui du segment auquel il appartient. La pirogue contenant les crânes ne peut être considérée comme un reliquaire. Cependant son emplacement est significatif. Selon Mercier (1952: 13), le crâne de chaque roi d'Abomey était conservé dans une case à l'intérieur du palais: "là se dresse leur jeho, case spéciale, ronde ou ovale, où a été placé leur crâne retiré du tombeau et où ils sont censés résider après leur mort. C'est devant cette case que l'on plante les asẽ, dissimulés par un voile: c'est là que l'on fait les sacrifices et les libations. C'est au point de vue religieux la partie centrale des coutumes. On voit apparaître ici une relation très forte entre crâne et asẽ. Les deux manifestent la présence du roi mort sous des formes différentes: crâne caché, asẽ-autel exposé à la vue du peuple. Pendant la cérémonie des coutumes, les asẽ sont portés au marché, placés dans une enceinte de nattes sur la place simboji [devant le palais royal]". 


\section{Remarques:}

- Traitement des crânes: lieux et parcours

Le corps, séparé de la tête, pourrit dans la maison, retourne au $\mathrm{cru}^{48}$, à la terre dont il est issu. Les différents crânes sont transportés de la maison de chaque mort à la xweta, "tête de maison", centre du quartier, où ils sont rassemblés dans une salle à proximité de celle de l'ancêtre fondateur. Là, chaque crâne placé dans une petite jarre déposée dans une grande jarre de terre cuite est transformé en un corps bien cuit ${ }^{49}$ habillé et honoré. Le processus d'ancestralisation des morts passe par ce lieu proche du sanctuaire. Mais ce rapprochement est encore marqué par de la distance: les morts ne sont pas introduits dans le sanctuaire des ancêtres à qui on rend un culte régulier. En fait, ils sont encore à mi-chemin entre les vivants et les ancêtres: d'un côté, on leur offre leurs mets préférés et on les conduit au marché comme s'ils étaient vivants et de l'autre on leur fait des sacrifices comme s'ils étaient déjà des ancêtres. Cette position intermédiaire est encore accentuée lors de l'"embarquement" des crânes: au moment où ils sont

48 L'opposition terre crue/terre cuite est soulignée par nos informatcurs. Sclon Guédou, lorsqu'un homme offense son " $j o t s$, son ancêtre protecteur, celui-ci lui lance de la terre erue; "au contact de celle-ci, son corps qui est de 'terre pétrie cuite', se transforme en terre crue. Cela entraine la décomposition physique et morale de la personne (1985: 374).

${ }^{49}$ Ce dispositif est remarquable. A partir du crâne (taka, "calcbasse de la tête" ou taxu, "os de la tête"), c'est la tête, puis le corps tout entier qui sont reconstitués. La tête est à la fois la partie la plus sacrée et le tout du corps. Durant la vie, la tête est le lieu du souffle et du destin individuel et lignager de chaque personne. Dans l'idéologie yoruba, on choisit son destin en choisissant sa tête (cf. P. Verger, 1981: 61 et Abimbola, 1981: 73-91). Celle-ci est considérée comme un vodun. Selon B. Maupoil (1961: 441), "celui que la fortune a favorisé doit sacrifier en l'honneur de sa tête considérée comme demeure d'un principe divin. Le devin fait trois onctions sur les trois parties essentielles de la tête: milieu du front, sommet de la tête et la nuque, puis sur d'autres parties du corps". On voit ici que le sacrifice à la tête concerne le corps tout entier. Maupoil (1961: 440) décrit un sacrifice de cabri fait en cas de maladie grave. La tête du cabri est mise en contact avec la tête du malade qu'elle remplace vis-à-vis de la mort, puis le sang du cabri versé sur des feuilles est mis à cuire sous les trois pierres du foyer. On dit que la tête du malade se trouvera alors cuite et recuite. Traiter la tête c'est traiter la totalité du corps, de même traiter le crâne c'est traiter le corps dans son côté inaltérable et le renvoyer au pays des morts. La tête comme le vodun ne meurt pas mais perdure en rejoignant le monde des morts. 
envoyés vers le pays des morts, ils sont mis à distance des vivants mais aussi des ancêtres du sanctuaire puisqu'on efface les traces de leur départ.

\section{- Les enveloppes}

Cette fois, les enveloppes ne sont ni enterrées ni brûlées mais exhibées de deux manières: vêtements neufs pour les ayisun, vêtements cérémoniels des morts portés par les enfants sur le marché. Ces enveloppes marquent la présence individualisée des morts parmi les vivants.

\section{- Le rapport morts/vivants}

Lors de cette seconde cérémonie d'ayisun, on a bien rassemble les différents crânes, mais la mobilisation du lignage n'est pas une simple addition des actes rituels accomplis par les différentes maisons. Dorénavant et pour toute la suite des cérémonies, le lignage en tant que totalité se manifeste à travers deux personnages importants: le xweduto, chef du lignage, et la tanyinon, prêtresse de l'ancêtre fondateur. Le premier doit se rappeler le nom de tous les morts, la seconde, qui est l'officiante principale de ces cérémonies, purifie, met les crânes dans les jarres, fait les sacrifices et embarque ces corps "bien cuits" pour le voyage vers le pays des morts. Elle agit pour les vivants au nom des ancêtres.

Il faut aussi rappeler que les membres du lignage doivent se réconcilier avant de commencer cette cérémonie et qu'ils demandent à ces morts, comme ils le font aux ancêtres, la prospérité (progéniture, bonnes récoltes, etc.) pour tout le groupe. La participation des gendres et des "enfants de filles" montrent que les liens de mariage sont aussi renforcés par cette cérémonie. Dans le mouvement même qui la manifeste, la totalité lignagère se présente donc comme ouverte vers l'extérieur.

Alors que les premières funérailles et les deux cérémonies ayisun traitent les morts à travers le corps (le cadavre puis le crâne), la cérémonie qui suit va concerner les défunts sous la forme d'une respiration, d'un souffle vital. 
2. La cérémonie cis wiwo, "attraper les morts" 50

Le nom de cette cérémonie vient de cis qui désigne le défunt et wiwo, terme d'origine yoruba qui signifie "action de tirer vers soi", "attraper". Il s'agit donc d'attraper les défunts, en fait de capturer Is gbigb $^{51}$, la "respiration", le "souffle" des morts. Ce rite commence trois jours après ayisun pour les hommes et cinq jours après pour les femmes.

\section{- Capturer les souffles à un carrefour}

Un cortège s'organise. Les représentants de chaque famille d'un mort portant une natte kplakpla, précédés de la tanyinon tenant deux calebasses, l'une remplie d'eau, l'autre de bouillie de mil, vont à un carrefour situé à la périphérie du quartier. Là, des tas de sable (autant qu'il y a de morts à invoquer) ont été préparés ${ }^{52}$. La tanyinon verse sur chaque tas de l'eau et de la bouillie de mil en disant: "Nous vous invitons à revenir dans votre foyer pour participer aux cérémonies que nous ont recommandées les ancêtres; accordez-nous beaucoup de bonheur, de prospérité, de progéniture". Le délégué de la famille pose sa natte kplakpla a cheval sur un tas. La tanyinon ou l'un de ses

${ }^{50}$ Chez les Fon, on ne pratique pas le rite d'ayisun, mais un rite semblable à $c i$ wiwo, appelé ci do hũ qui signifie "embarquer le mort". A Porto Novo on appelic aussi ce rite ago. Comme dans le rite ago vive, il s'agit de traiter les restes actifs des morts, ici le souffle sous la forme de tessons de poteries.

51 Le gbigbo durant la vie est la respiration, le souffle. A la mort il quitte le corps et devient une "âme" errante invisible, dangereuse. Dans ce rite, il s'agit de capturer celle-ci sous la forme de souffle à l'appel de son nom, de lui redonner en quelque sorte une vie individualisée.

$52 K J$ désigne la terre, le sable, la matière première avec laquelle le corps matériel de l'homme est pétri. Selon Pazzi (1976: 62) "à la mort, le corps se décompose et se disperse, on dit alors ezù $k o$ (il est devenu sable)". Mais il semble bien que le sable représente aussi le yé du mort, son ombre, son âme. Rappelons que quand le mort est enterré dans le cimetic̀re, on rapporte du sable du dessus de la tombe pour l'ensevelir dans la maison. Chez les Fon, où les femmes mariées sont enterrées dans la maison de leur mari, on va chercher sur leur tombe de la terre que l'on ramène cérémoniellement dans la maison de leur père pour procéder au rite de ci do hsn, appel et transformation des morts (Adoukodonou, 1979: 164). Ce sable est transporté dans une poterie sur le "chemin du $s \varepsilon^{\prime}$ (chemin du destin). 
assistants appelle les morts un par un: "Un tel, viens boire de l'eau dans la maison de ton père" 53 . A la fin de l'invocation, on sent un grand vent ou un froid glacial. Le délégué se précipite pour fermer solidement sa natte kpakpla capturant ainsi le souffle du mort. Le cortège prend le chemin du retour. Chacun porte sa natte sur la tête et marche en la balançant (on dit que c'est le gbigbs, le "souffle" qui produit ce mouvement) et en chantant: $Z \delta$ na ma le, ajivi vulo, gbe jots, zo na ma le, "Marche que je te suive, l'enfant ne refuse pas la maison où il est né, marche que je te suive". Arrivé sur une petite place (proche du sanctuaire de l'ancêtre) consacrée pour la circonstance $^{54}$ et appelée place d'ago, on dépose les nattes par terre. Le cinquième jour, on fait la même cérémonie pour les femmes.

- Casser les pots, rassembler les souffles et les honorer sur la place d'ags

Le lendemain, la famille de chaque défunt apporte un agoze ${ }^{55}$, un pot en terre qui représente le souffle du mort. La tanyinon prend le premier pot et, tenant un bord avec la main gauche, elle appelle le souffle du défunt en le nommant: "Un tel, nous faisons pour toi ce que nous avons fait pour tes aïeux, ce que tes aïeux nous avaient enseigné; nous t'invitons à prendre part à cette fête...". Après cette invocation, la tanyinon et le nonuvi ("enfant de fille"), qui tenait de la main gauche l'autre bord de la poterie, la laissent tomber et se briser sur le

53 Il faut noter que l'on appelle aussi les mauvais morts, les morts dans la brousse, les morts à l'étranger. Il est très important que tous les souffles reviennent dans l'enclos parental.

54 Deux nattes kplakpla ont été enterrées et recouvertes de terre aplanie. C'est le rappel de la tombe d'un mort.

55 Dans certains lignages, on capture le souffle dans le pot qu'on enferme ensuite dans la natte kplakpla. Herskovits (1967: I, 198-199) décrit un rite du même type où le souffle du mort est représenté par un poussin: "He [l'officiant] throws the chick inside the loboze, closing it immediately with his hand, since the chick acts as a substitute soul for the summoned". A la fin de l'appel des morts, l'officiant reçoit quarante et un poussins de plus: "He kills these, tearing them to pieces, throwing the meat among the group of small pots". Le même rite est décrit par Adoukonou (1979: 149). 
sol. On fait de même avec les autres agozin. Puis les deux officiants rassemblent, avec la main gauche, les tessons en un tas. Trois tas de souffles sont ainsi constitues: un pour les hommes, un pour les femmes et un pour les défunts dont on aurait oublié le nom. Un abri est dressé au-dessus de chaque tas. Pendant huit jours, les parents des défunts leur apportent à manger, leur offrent des libations et des sacrifices par l'intermédiaire de la tanyinon. Comme lors de la deuxième cérémonie d'ayisun, le cinquième jour est celui des libations et des sacrifices offerts au nom de tout le lignage.

- "Ouvrir les cassures"56, renvoyer les souffles: le sacrifice du porc

Le soir du huitième jour, on procède au sacrifice d'un jeune porc ${ }^{57}$ et à l'ouverture des "cassures" des pots par la tanyinon. Toutes les familles cotisent pour l'achat de ce jeune porc qui est appelé ago kikija hã, "porc pour disperser ago". Un descendant du lignage (sunuvi) ou un enfant des filles (ñonuvi) présente l'animal à la tanyinon puis le sacrifie. Il verse du sang sur les differents tas. On dit dans ces circonstances: $N u$ kpevi we nogba nu daxo, "c'est une petite chose (le jeune porc) qui disperse habituellement une grande chose".

La tanyinon portant autour du cou des feuilles de palme (aza) verse de l'huile de palme pour diminuer la force du sang du porc ${ }^{58}$, puis une calebasse de céréales (haricots, maïs) en disant: "Nous achevons ago dans le bonheur, la prospérité, la paix, épargnez-nous les morts subites". En versant l'eau, elle ajoute: "Nous entendons vivre désormais dans la fraîcheur, que tous les enfants présents ou absents soient en bonne santé; maintenant que nous avons rempli nos obligations envers vous, emportez nos peines et nos souffrances". En jetant

56 C'est-à-dire ici séparer les tessons qui sont agglutinés. Mes informateurs disent en français "cassures" au lieu de tessons. Ce terme me semble très suggestif car il met en valeur l'acte mème de casser.

${ }^{57}$ Les animaux sacrificiels courants sont le coq, la poule, le cabri, la chèvre. Pourquoi, dans cette circonstance, choisit-on le pore? Selon un informateur de Le Hérissé (1911: 169) "Le porc a une chair qui ressemble à celle des cadavres". Faut-il en conclure que manger le porc, e'est manger le ou les morts ?

58 On dit que le sang excite les morts et que l'huile de palme les calme. 
ses colliers d'azã sur les tas d'ago, elle fait des voux pour la collectivité et pour elle-même: "Comme toutes les tanyinon qui m'ont précédée, j'ai officié dans la franchise et l'amitié, sans rancour ni rancune; je prie tous les ancêtres (kuvits) dont la fête vient d'être célébrée de protéger la collectivité, de donner beaucoup de yao (brus), d'enfants, de faciliter le commerce, que moi-même je sois gardé par vous en bonne santé jusqu'aux prochaines cérémonies". Elle ouvre alors quelques "cassures" d'ago de la main gauche. Les assistants l'imitent. Le porc est ensuite préparé et cuisiné (sans sel dans certains lignages). Tous les orphelins doivent en manger. On garde les os pour les absents. Ce dernier repas funéraire les libère définitivement des interdits liés au deuil. La soirée se termine par les chants et les danses au son du tam-tam funéraire. Au petit jour, on prépare le départ d'ag ${ }^{59}$.

\section{La cérémonie ago, brâler les restes des morts dans la brousse.}

Les "cassures" sont enveloppées dans plusieurs nattes kplakpla. Dans un des paquets, on met le crâne du porc. On prépare les bagages ${ }^{60}$ des morts : vêtements de cérémonies, ustensiles de cuisine et de travail ${ }^{61}$. Un cortège s'organise: en tête trois personnes avec des

59 Les rites décrits ici sont ceux que nous avons observés dans plusieurs lignages de Porto-Novo. Kiti (1968: 17-19) décrit des rites similaires dans la banlieue de Porto-Novo. Il faut noter une différence importante. Le lendemain de l'appel des morts, ceux-ci sont accueillis dans le temple de l'ancêtre sous forme d'asẽ (on ne dit pas combien, est-ce un asẽ par catégorie ?). On sacrifie une truie ayant eu des petits dont on verse le sang sur les asé. Puis on cuisine certaines parties de l'animal. Quelques bouchées sont déposées sur les asẽ, "c'est ainsi qu'on donne à manger au kuvit morts". Ensuite chaque assistant réclame une petite part.

${ }^{60}$ Agban désigne les bagages en général, mais aussi les cadeaux, en particulier la compensation matrimoniale. Bo agban signifie déménager, ramasser ses affaires. A la fin de la cérémonie du retour provisoire des ancêtres royaux (nonsu xwe), Herskovits (1967: I, 227) décrit le dernier rite précédant le départ de ces ancêtres: "packing up the 'luggage' of their ghostly masters".

61 Dans le rite observé par Kiti (1968: 19-20), ce sont les balayures de toutes les maisons qui sont entassées dans les nattes kplakpla et brûlées à ag avec les offrandes de la famille: beaux pagnes, alcool, vaisselle neuve. 


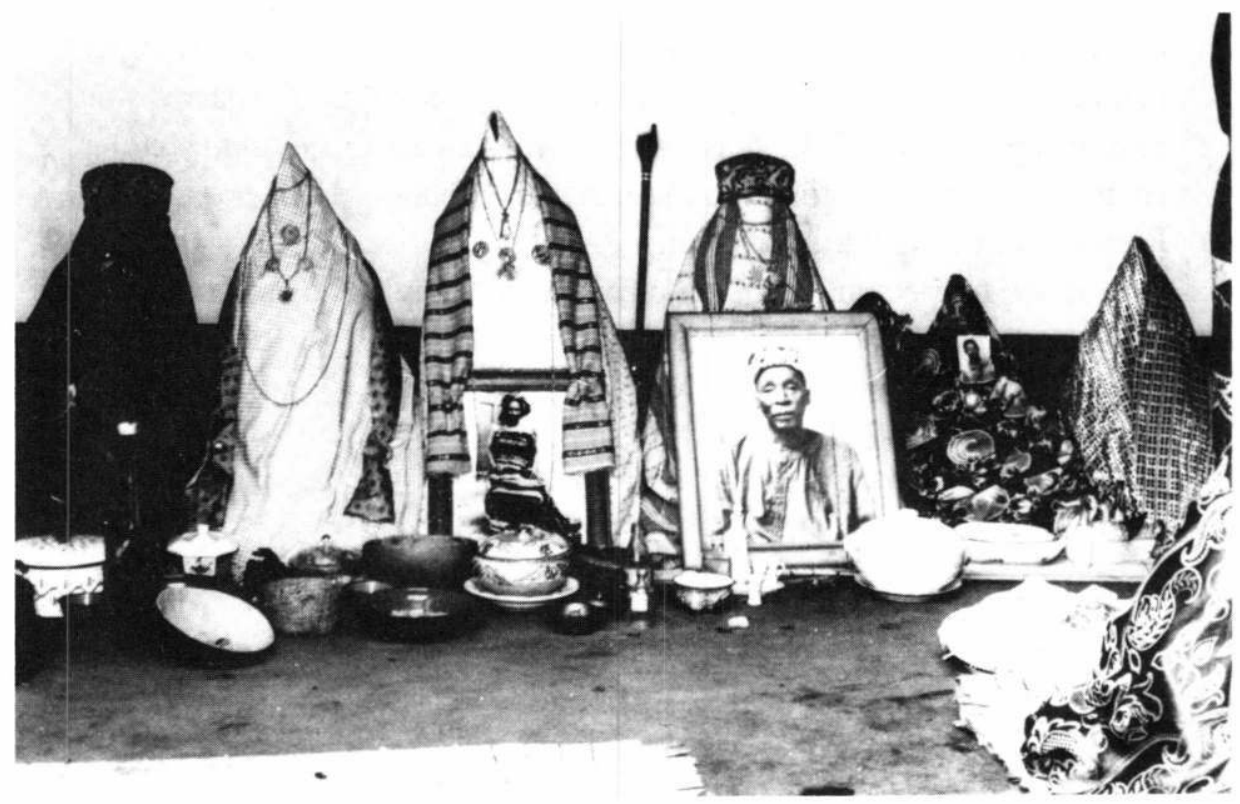

Exposition des ayisun 
drapeaux rouge, blanc et noir ${ }^{62}$, ensuite les porteurs des paquets de "cassures" (les enfants de filles - les nonuvi - dans certaines familles, les veuves dans d'autres), puis les porteurs des bagages, enfin la tanyinon suivie des membres du lignage. La procession cérémonielle est accompagnée par des tam-tam de dérision (on tape sur toutes sortes de récipients en fer) et des chants d'adieu aux morts. Tout le long du cortège, des hommes brandissent des armes, des coutelas ${ }^{63}$. Après un arrêt sur le marché, on se dirige vers la brousse en chantant: "Nous allons les accompagner, nous allons les conduire". Arrivés à agoto, lieu d'ags dans la brousse, un feu est allumé dans lequel les nattes, les cassures et tous les bagages sont jetés. Seuls les nonuvi, enfants de fille, peuvent s'emparer des objets des morts; ce vol rituel est bénéfique pour le lignage de leur oncle maternel. Pendant que le feu brûle, on jette des pièces de monnaie, des assiettes neuves pour honorer les défunts. Ceux-ci sont supposés emporter dans l'au-delà tous les objets consumés par le feu. Quand celui-ci commence à s'éteindre, la tanyinon verse une dernière libation d'alcool et d'eau et toute l'assistance tourne autour en chantant des chants funèbres puis repart en cortège se purifier dans la lagune avant que le feu ne s'éteigne.

Les cérémonies ayisun et cis wiwo traitent les morts sous des formes différentes: le crâne, le souffle. Dans les deux cas, les morts sont rassemblés dans un lieu consacré proche du sanctuaire de l'ancêtre où ils sont ensuite renvoyés. Mais les parcours et les traitements diffèrent.

${ }^{62}$ Ces trois couleurs manifestent l'ambivalence de la mort et des sentiments qui lui sont liés. Le noir est lié à la souffrance, au deuil (les pagnes de deuil sont bleu-noir), le blanc se réfère à l'au-delà, aux ancêtres (la tanyinon porte un pagne blanc lorsqu'elle officic), il est signe de joie. Le rouge est la couleur du sang, de la violence mais aussi celle de la vie. Le blanc et le rouge sont associés à l'orient, le noir à l'occident (Pazzi, 1967: 37).

${ }^{63}$ Que signifie la présence de ces hommes armés ? Selon nos informateurs, ils sont là pour écarter les parents et assistants qui voudraient s'emparer des affaires des morts. Cette explication nous paraît insuffisante. En effet, si ces restes des morts ne sont pas brûlés, ceux-ci risquent de venir hanter les vivants et leur apporter la maladie et la mort. Cependant, il nous semble que la menace des armes s'adresse aussi aux morts: il s'agit de les chasser du monde des vivants. 
Le traitement des crânes commence à la maison, continue près du sanctuaire de l'ancêtre où ils sont reconstitués comme corps bien cuits puis "embarqués" collectivement tout en restant bien individualisés, à destination de l'au-delà de la mer, le monde des morts. Les vivants ne quittent jamais le quartier.

Les souffles sont captés à l'extérieur du quartier à un carrefour puis ils sont amenés à la place d'ag., proche du sanctuaire de l'ancêtre, où ils sont traités. Que signifie l'appel des souffles au carrefourmarché ? Celui-ci est le lieu où reviennent les morts errants. Appeler, siffler les noms des morts, c'est leur redonner le souffle, la respiration, le gbigbs, c'est ranimer la relation individuelle de chaque mort avec les vivants. Ce moment est ressenti comme très dangereux par les parents qui tournent le dos au moment de l'appel et s'empressent d'emprisonner le gbigbo dans la natte kplakpla.

Ces gbigbo sont traités sur la place d'ags. Le petit pot (qui représente le gbigho) est cassé violemment. Par ce geste violent, on coupe la relation entre le mort individuel et son souffle de vie. Rassembler les "cassures" des pots par catégories, c'est rassembler les souffles dans leur relation à l'ancêtre par l'intermédiaire de la tanyinon. On honore les souffles en tant que fils et filles de l'ancêtre ${ }^{64}$. Le renvoi des morts se fait par le sacrifice du porc et par l'ouverture des "cassures". Le sacrifice du porc unique offert par toute la collectivité du lignage constitue le moment de la plus grande proximité entre les vivants et les morts représentés sous forme de tas: les premiers mangent la chair cuite de l'animal alors que les seconds reçoivent son sang. Mais c'est aussi le moment de la séparation, celui qui permet l'expulsion, le départ de ces souffles par l'ouverture des cassures. Les morts sont devenus des kuvito ${ }^{65}$, c'est-à-dire des ancêtres non

\footnotetext{
${ }^{64}$ Selon Adoukonou (1979: 143), par ce rite on retire les morts de l'état d'oubli et de souffrance pour les intégrer dans l'enclos familial avec l'ancêtre afin qu'ils veillent sur le lignage, les enfants, les femmes.

65 Nous traduisons ce terme par "ancêtre", mais il ne faut pas oublier son ambiguité. Selon Ségurola, il désigne les morts dans l'au-delà, les revenants et les morts auxquels on fait des offrandes sur les asẽ. Il faut noter que ce terme est employé le plus souvent au pluriel: on fait un culte aux kuvits, on consulte les kuvits, etc.
} 
nommés mais placés sous le contrôle de l'ancêtre fondateur. Alors que dans la cérémonie des crânes, les défunts sont renvoyés dans le pays des morts sous une forme individualisée, ici les souffles sont traités par catégories et renvoyés collectivement dans l'anonymat ${ }^{66}$. Dans cette cérémonie, on a coupé les morts de leurs relations aux vivants, on les a placés dans la relation à l'ancêtre, on les a transformés en kuvito, fils et filles de l'ancêtre, des pourvoyeurs de vie comme le dit bien la prière de la tanyinon.

Une fois les souffles partis, il ne reste plus que les cassures et aussi tous les habits et objets des morts, l'ensemble ne formant que des enveloppes vides. Ce sont ces traces sans vie que l'on doit brûler dans la brousse: il s'agit, d'une part, d'envoyer dans le pays des morts, des objets, des enveloppes qui permettront aux défunts de tenir leur rang, leur statut, et, d'autre part, d'effacer tout ce qui est susceptible de permettre un retour possible des morts ${ }^{67}$. La place d'agoto où se fait cette dernière cérémonie est une sorte de double de celle qui se trouve à côté du sanctuaire de l'ancêtre. Elle est dans les deux cas un lieu consacré de transformation des morts. Mais alors que dans le quartier, elle rapproche les morts du sanctuaire de l'ancêtre, dans la brousse, elle est ce lieu de passage entre le monde des vivants et le monde des morts, celui de l'origine.

Il faut aussi mettre en contraste le feu d'ago des premières funérailles, rite d'expulsion, rite de rupture, avec ce second feu, rite sacrificiel où la mise en relation des morts avec l'au-delà prend le pas

${ }^{66}$ Ici se pose un problème que nous ne pouvons résoudre: où partent les souffles ? Selon les uns, ils vont rejoindre les ancêtres au pays des morts (Akoudonou, Herskovits). Mais on dit aussi qu'ils doivent retrouver le créateur, le grand Se ou Mawu, pour donner naissance à d'autres vivants. Ils sont censés revenir dans le lignage par l'intermédiaire du joto, de l'ancêtre qui va chercher l'argile dont sera pétri le corps (Guédou, 1985: 320-330).

${ }^{67}$ Dans un article sur les funérailles des Batammariba du Nord Togo, M. Smadja (1988: 74) désigne l'ensemble des objets jetés ou enterrés sous le terme "les affaires du mort" qui comprennent d'une part les effets personnels du mort, "prolongement du corps", de l'autre "les affaires de mort", objets qui ont représenté celui-ci à un moment du rite. On retrouve ici le même ensemble mais traité différemment, c'est-àdire brûlé. 
sur l'expulsion. Plutôt qu'une rupture, ce dernier feu manifeste l'apothéose de la transformation des morts en ancêtres - encore anonymes qui vont rejoindre leurs peres.

Dans tous ces rites, il faut noter le rôle central joué par la tanyinon: elle appelle les morts au carrefour, elle casse le pot et quelques jours plus tard ouvre les cassures qui liberent les souffles, enfin elle met le feu aux restes des morts. Lors de certains actes rituels, elle est secondée par les nonuvi, "enfants de fille" qui apparaissent là comme son prolongement.

Après l'enterrement du cadavre, l'embarquement des crânes, la capture puis la libération des souffles, enfin l'embrasement des enveloppes, toute trace de caractère vivant de chaque mort est effacée. Mais aussi toute trace de leur individualité a disparu: les morts récents sont devenus des kuvits, des ancêtres anonymes. Tant que leur nom n'a pas été fixé, planté, ils restent dangereux. On attend pour faire le dernier rite de fixation des kuvits que l'un d'eux ait réclamé un sacrifice par l'intermédiaire du fa. Alors les familles des morts se mobilisent pour acheter des asẽ et préparer la cérémonie.

4. La cérémonie asẽyidota : planter le nom du mort dans le sanctuaire de l'ancêtre fondateur

Le terme qui désigne cette cérémonie est composé de asẽ "parasol", nyi, "nom" et dote, "être droit", "être debout". Il s'agit de planter, de "mettre debout" les parasols nommés représentant les morts transformés en ancêtres dans le sanctuaire de l'ancêtre fondateur.

Cetie cérémonie peut avoir lieu juste après la fin de la cérémonie d'ago ou plusieurs années après. Elle est collective. Chaque famille achète au marché un asê, un parasol pour son mort qui n'a pas encore été fixé. La veille au soir les asẽ sont rassemblés puis lavés dans une mixture d'eau et de feuilles liturgiques devant le sanctuaire de l'ancêtre. A l'intérieur de celui-ci, la tanyinon prépare un endroit où elle verse le reste des feuilles macérées.

Le matin la tanyinon traîne chaque asẽ dans le sanctuaire en nommant le mort. Elle invoque la série d'ancêtres qui l'ont précédé en commençant par le plus ancien pour arriver jusqu'au plus récent et 
quand elle arrive au mort lui-même, elle plante l'asẽ dans le sol en prononçant son nom ${ }^{68}$. Elle entoure ensuite l'asẽ d'une natte kplakpla.

Le lendemain dans l'après-midi, les familles des morts se réunissent devant les nouveaux ase. La tanyinon dit des prières, enlève les nattes $k$ plakpla ${ }^{69}$, fait des libations et des sacrifices de poulet et de cabri apportés par les orphelins et les gendres. On clôt la cérémonie par la danse au son du tam-tam appelé kete. Des louanges sont chantées pour les nouveaux morts.

Pendant cette cérémonie, on reste dans le quartier, mais chaque asẽ est traîné de l'extérieur vers l'intérieur du sanctuaire. Comme lors de la cérémonie des crânes, le mort est individualisé par son nom et le parasol. Mais il y a aussi des différences importantes : l'asẽ représente son nouveau statut d'ancêtre. Pour les vivants, le parasol est le signe d'un statut de dignitaire lors des parades cérémonielles. Pour les morts, il est planté et associé à un nom et à une généalogie. Le prestige plus ou moins grand des morts se manifeste dans la décoration des ase et dans les louanges qui leur sont chantées ${ }^{70}$.

Tous ces ase sont placés autour de celui de l'ancêtre fondateur. Il existe une sorte de débarras dans lequel sont remisés les vieux ase dont on a oublié le nom. La généalogie récitée pour chaque maison ou

${ }^{68}$ Il ne s'agit pas de toute la chaîne généalogique des ancêtres mais de ceux qui ont marqué l'histoire du lignage et des plus récents dont on a gardé le nom.

${ }^{69}$ Notons la place spécifique qu'occupe la natte kplakpla tout au long des rites funéraires. Durant les premières funérailles, dans la tombe, les nattes entourent le mort pour l'isoler de la terre comme elles séparent le mort du reste de sa famille, de sa maison. Seuil, frontière, limite, les nattes séparent le monde des vivants de celui des morts. On enlève définitivement la natte kplakpla au moment où l'on offre la première libation à l'ancêtre nommé, moment où sa relation aux vivants est rétablie. Cette natte est présente dans d'autres rites du quartier. Toute future initiée au vodun Sakpata (vodun de la terre) est dite dans un premier temps tuée par celui-ci. Elle s'abat par terre comme morte, et est enroulée dans des nattes kplakpla pour être emportée dans le couvent de l'initiation.

${ }^{70}$ Les asẽ sont des parasols en métal. Ceux des morts les plus importants sont parés de pendentifs et surmontés de symboles rappelant les hauts faits et les paroles du défunt qui sont chantés dans leurs louanges. Ils manifestent done le prestige social de l'ancêtre. Ils sont en même temps des autels sur lesquels on offre à manger et à boire aux ancêtres. 


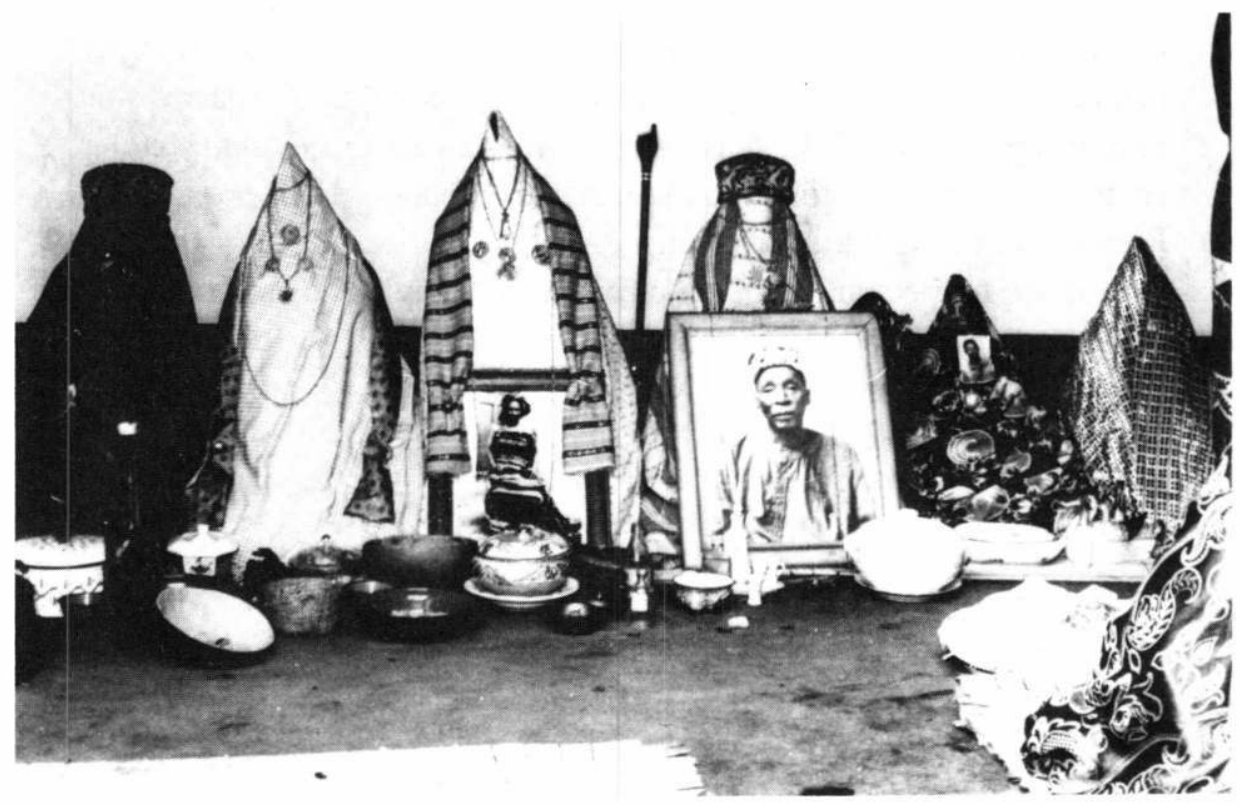

Exposition des ayisun 
segment de lignage ne retient des morts que le père, le grand-père, l'arrière-grand-père du plus âgé des vivants, et enfin l'ancêtre fondateur du segment ou du quartier. Parfois l'arrière-grand-père peut être remplacé par un ancêtre plus lointain qui a joui d'un prestige particulier. Les ancêtres intermédiaires ne sont pas remémorés. Il en est de même pour les asẽ. Seul l'ancêtre apical n'est jamais oublié, c'est lui qui établit les ancêtres intermédiaires dans leur statut. Ceux-ci se mettent sous sa protection, s'identifiant à lui en quelque sorte, renouvelant la relation à l'origine.

\section{Conclusion}

Planter l'asẽ du mort est l'aboutissement du long processus du traitement du nom ${ }^{71}$. Durant toutes les secondes funérailles, le nom de chaque défunt est invoqué, associé aux composantes du mort que l'on traite. Dans la cérémonie du cis wiwo, nommer prend une force particulière: crier, siffler le nom, c'est redonner le souffle, la respiration à chaque mort, c'est le rendre présent sous sa forme la plus dangereuse, celle d'un mort errant, d'un mort trop vif. Casser les pots, c'est effacer l'individualité dangereuse de ces morts, c'est aussi dissocier le nom du mort des vivants, le rendre anonyme en l'intégrant à une même catégorie de souffle et le rapprocher des ancêtres. Les secondes funérailles peuvent donc être considérées comme un processus de transformation du nom, de sa séparation du corps matériel et du souffle vital du mort, comme une mise à distance progressive du mort et des vivants, comme un ensemble d'opérations qui établissent les

${ }^{71}$ Qu'est-ce que le nom ? Selon Guédou (1985: 324), le nom définit l'individu, il le crée et lui donne un statut social. L'individu change de nom chaque fois qu'i] change de statut. Guédou distingue les noms d'enfance déterminés par les circonstances de la naissance et les noms choisis: noms de jeunesse, noms de chef. Vers dixhuit ans, le jeune homme et la jeune fille se choisissent un nom, wen nyi, "nom de message". Ce nom est un programme de vie et la première parole sociale de l'individu. Il pourra en changer aux moments importants de sa vie: mariage, accès à un statut de chef, etc. Les louanges des ancêtres déclinent les noms forts que ceux-ci se sont attribués au cours de leur vie. 
morts dans une proximité avec l'ancêtre fondateur présent dans le sanctuaire.

Le nom est certes ce qui reste des morts envoyés vers l'au-delà des mers, mais e'est plus que la remémoration de leur passage, le souvenir de ce qu'ils ont été. Planter le nom des morts auprès de celui de l'ancêtre fondateur, c'est en quelque sorte régénérer celui-ci, le replanter; c'est rendre présent le temps de l'origine ainsi que l'écoulement des générations dans une structure spatiale, territoriale qui relie le sanctuaire aux différentes maisons du quartier. Les morts plantés sont ainsi placés entre l'ancêtre fondateur considéré comme l'aîné absolu, le père du lignage et les vivants, c'est-à-dire les cadets, les enfants du lignage. Le temps qui passe permet l'oubli des générations intermédiaires, mais non celui de la fondation, ou plus précisément de la distance entre l'origine et le present. Planter le nom des morts, c'est en définitive reconstruire le lignage dans son rapport au temps et à l'espace.

Il faut ajouter ici un fait significatif. A chaque naissance d'un enfant, on cherche parmi les ancêtres, celui qui est son jots. Ce terme qui signifie litteralement "naître-père" désigne l'ancêtre protecteur de chaque personne, "celui qui a cherché la terre dont on a fait le corps d'un individu"72. Généralement il s'agit du grand-père ou de l'arrière grand-père de l'enfant ou d'un ancêtre prestigieux, parfois même de l'ancêtre apical. Un des noms portés par une personne sera celui de cet ancêtre-jot $\supset$ et un petit asẽ le représentant sera placé auprès de l'asé de l'ancêtre. Ainsi dans le sanctuaire, les générations passées et présentes sont étroitement associées, l'inscription des morts comme ancêtres construit aussi la continuité du quartier.

Comme lors de la cérémonie des crânes, le mort est individualisé, mais cette fois, par l'asẽ qui porte son nom. Mais alors que le crâne

\footnotetext{
${ }^{72}$ Guédou (1985: 236). L'auteur ajoute: "Chaque homme doit connaître son jot s qui est en quelque sorte le fournisseur de la matière première dont il est fait. Lorsqu'un individu offense son $j \mathfrak{t} \supset$ ou commet une faute grave contre les défunts de son clan, la colère du jots peut le rendre malade. On dit alors que "son jots lui a jeté de la terre crue".
} 
est censé être parti aux pays des morts, l'asẽ représente le mort ancêtre résidant dans le quartier. Comme le crâne représente l'identité du mort dans l'au-delà, l'asẽ représente l'identité du mort parmi les vivants.

Qu'en est-il de l'opposition homme/femme ? Au moment du mariage, une femme quitte la maison de son père pour aller dans celle de son mari, où elle sera une yao, celle qui chante les louanges des ancêtres de son époux. Mais elle reviendra chez son frère après la ménopause pour jouer pleinement le rôle rituel de la tante paternelle, assistante de la tanyinon. Ce mouvement des femmes par le biais du mariage, les rites funéraires en conservent la trace, en marquent l'importance, puisque les nonuvi, les "enfants de fille" assistent la tanyinon lors des cérémonies et peuvent prendre une partie des biens destinés aux morts.

A leur mort, les fils et les filles du lignage reçoivent le même traitement rituel. Leur nom est planté dans le sanctuaire mais disparaîtra au bout de deux à trois générations. Seuls le nom de l'ancêtre fondateur - en tant que père du lignage et aîné absolu - et ceux des ancêtres à l'origine des segments cadets resteront inscrits sur les sanctuaires, les yoxo, qui structurent le quartier (cf. Pineau-Jamous, 1986). Tous ces ancêtres sont des hommes et manifestent le lignage dans son rapport à l'origine, au passé, à la durée. Cependant le rôle des femmes, en tant que tantes paternelles, tanyi, et plus particulierement celui de la tanyinon, est essentiel. Cette dernière est toujours choisie dans la lignée aînée, alors que le chef du lignage (xweduto) est choisi alternativement dans une des lignées cadettes. D'un point de vue structural, elle est une sœur aînée par rapport à ce chef. De ce point de vue, elle est en quelque sorte le double vivant de l'ancêtre fondateur, son porte parole, et possède le pouvoir de bénir ou de maudire en son nom. Ce qu'on retient, ce n'est pas le nom des tanyinon successives, mais le fait que des femmes issues de la lignée aînée, la lignée la plus prestigieuse, ont occupé et occuperont la même position rituelle.

$\mathrm{Au}$ terme de notre analyse se manifeste toute l'importance d'une femme, la tanyinon, la sour aînée, prêtresse des ancêtres. C'est elle qui transforme les morts en ancêtres, c'est elle qui inscrit aussi les 
enfants de ses frères dans le lignage lors des rites de passage. La filiation patrilinéaire est plus qu'une simple transmission de statut entre un pere et ses fils. Elle est plus qu'un engendrement biologique qui ferait intervenir la complémentarité du couple. C'est l'action de chaque tanyinon dans le présent rituel qui fonde la relation entre le passé et le futur, entre les ancêtres et leurs descendants agnatiques, c'est elle qui assure rituellement la continuité des générations. Elle est en quelque sorte la face cachée et active de la filiation unilinéaire.

Marie-Josée Jamous

\section{Références bibliographiques}

Abimbola, W.

1981 "The Yoruba concept of human personality" in La notion de personne en Afrique Noire, CNRS, 73-91.

Adoukonou, B.

1979 "Pour une problématique anthropologique et religieuse de la mort dans la pensée Adja-fon", in Bamunoba, Y. K. \& Adoukonou, B., in La mort dans la vie africaine, Paris, Présence Africaine, Unesco, 118-335.

Akindele, A. \& Aguessy C.

1953 Contributions à l'étude de l'histoire de l'ancien royaume de PortoNovo, Dakar, Institut Français d'Afrique Noire (Mémoire ${ }^{\circ}{ }^{25}$ ).

Bloch M. et Parry J. (éd)

1982 Death and the Regeneration of Life, Cambridge, Cambridge University Press. 
Bourgoignie, G. E.

1972 Les hommes de l'eau, ethno-écologie du Dahomey lacustre, Paris, Editions Universitaires.

Glélé, M.A.

1974 Le Danxome, Paris, Nubia.

Guédou, G. A. G.

1985 Xo et Gbe, langage et culture chez les Fon (Benin), Paris, SELAF.

Herskovits, M. J.

1967 Dahomey. An Ancient West African Kingdom, Evanston, Northwestern University Press, 2 vol. ( $1^{\circ}$ éd. 1938).

Kiti, R. P. G.

1968 "Rites funéraires usités chez les Alladanous et diverses tribus de race Goun sur Aizo habitant la banlieue de Porto-Novo", IRAD, Etudes Dahoméennes (Nouvelle série), 11, 5-22.

Le Herissé, A.

1911 L'ancien royaume du Dahomey. Mcurs, religion, histoire, Paris, Larose, $384 \mathrm{p}$.

Maupoil, B.

1961 La géomancie à l'ancienne Côte des Esclaves, Paris, Institut d'Ethnologie, XXVIII, 690 p. (Travaux et mémoires $n^{\circ} 42$ ).

Mercier, P.

1952 Les asen du Musée d'Abomey, Dakar, IFAN.

Mondjannagni, A. C.

1977 Campagnes et villes au sud de la République du Bénin, Paris - La Haye, Mouton.

Pazzi, R.

1976 L'homme - eve, aja, gen, fon - et son univers, Dictionnaire. Lomé, (fascicule ronéoté).

Pineau-Jamous, M. J.

1986 "Porto-Novo: royauté, localité et parenté", Cahiers d'Etudes Africaines, XXVI, 4, 547-576.

Quenum, $H$.

1983 Au pays des Fons. Us et coutumes du Dahomey, Paris, Maisonneuve et Larose. 
Segurola, R. P. B.

1963 Dictionnaire Fon-Français, Cotonou (fascicule ronéoté).

Smadja, M.

1991 "Les affaires du mort" in Le deuil et ses rites, Systèmes de Pensée en Afrique Noire, XI, 57-89.

Tidjani, S. A.

1952 "Notes sur le mariage au Dahomey", Etudes Dahoméennes, VII, 7-79.

Verger, P.

1954 Dieux d'Afrique, Paris, Hartman.

1981 "Notion de personne et lignée familiale chez les Yoruba" in La notion de personne en Afrique Noire, Paris, CNRS, 61-73. 